\title{
Performance of Digital Back-Propagation in Kramers-Kronig Direct-Detection Receivers
}

\author{
Z. Li ${ }^{1}$, L. Galdino ${ }^{1}$, T. Xu' ${ }^{2}$, M.S. Erkılınç${ }^{1}$, K. Shi1 ${ }^{1,3}$, E. Sillekens ${ }^{1}$, B.C. Thomsen ${ }^{1,3}$, P. Bayvel ${ }^{1}$, and R.I. Killey ${ }^{1}$ \\ ${ }^{1}$ Optical Networks Group, Department of Electronic \& Electrical Engineering, UCL (University College London), London, UK \\ ${ }^{2}$ School of Engineering, University of Warwick, Coventry, UK, ${ }^{3}$ Microsoft Research Ltd, Cambridge, UK \\ E-mail: zhe.li@ee.ucl.ac.uk
}

\begin{abstract}
We report the first investigation of fiber nonlinearity compensation by digital backpropagation in Kramers-Kronig direct-detection receivers. Improvements in performance of $112 \mathrm{~Gb} / \mathrm{s} / \lambda \mathrm{WDM}$ transmission over single-span SSMF links of up to $160 \mathrm{~km}$ are demonstrated. OCIS codes: (060.0060) Fiber optics and optical communications; (060.2360) Fiber optics links and subsystems
\end{abstract}

\section{Introduction}

Metro and data center interconnect (DCI) networks are experiencing unprecedented traffic growth, requiring large numbers of low-cost, high-throughput optical transceivers operating at $\geq 100 \mathrm{~Gb} / \mathrm{s} / \lambda$ over single-span links $(\geq 80 \mathrm{~km})$ without inline amplification. With the ongoing advances in digital CMOS technology, direct-detection (DD) transceivers employing single-sideband (SSB) signaling and digital receiver linearization [1-4] potentially meet these requirements while using simple optical hardware structures. One recently developed receiver linearization technique, the Kramers-Kronig (KK) scheme [2-6], is particularly effective in removing the signalsignal beat interference (SSBI) due to square-law detection and accurately reconstructing the transmitted optical SSB signal in the digital domain.

In long single-span links, requiring high optical launch powers, fiber Kerr nonlinearity imposes a limit on the system performance. Digital back-propagation (DBP) is an effective approach to overcome this [7], but requires detection of the amplitude and phase of the optical transmitted signal. In direct-detection transceivers in which the phase of the signal is lost after detection, receiver-based DBP is ineffective. Transmitter-based DBP can be used [8], but operational complexity is increased due to the required prior knowledge of the link at the transmitter. However, employing the KK scheme, which carries out a linear mapping of the optical signal into the digital domain, should allow the use of receiver-based DBP after direct detection. The achievable gain with DBP in such receivers is an open question. The KK scheme requires an ideal single-sideband minimum-phase optical signal to achieve optimum performance [2]. However, distortion due to fiber nonlinearity may result in these conditions no longer being met, for example, due to growth of the unwanted sideband from four-wave-mixing between the optical carrier and the wanted sideband. In this paper, we address this question. A numerical study was first carried out, comparing the performance of DBP in Kramers-Kronig and ideal linearized receivers. Following this, system performance was assessed experimentally in a $50 \mathrm{GHz}$-spaced $4 \times 112 \mathrm{~Gb} / \mathrm{s}$ WDM SSB 16-QAM Nyquist-subcarrier modulation (Nyquist-SCM) DD system [3] transmitting over single-span standard single-mode fiber (SSMF) links up to $160 \mathrm{~km}$.

\section{Simulation of the Kramers-Kronig Direct-Detection Receiver with Digital Back-Propagation}

The receiver design including the KK scheme with either electronic dispersion compensation (EDC) or DBP is shown in Fig. 1(a). The transmitted single-polarization SSB signal is amplified by an erbium-doped fiber amplifier (EDFA) and the channel of interest is selected using an optical band-pass filter (OBPF). After square-law detection with a single photodiode, the resulting electrical double-sideband signal (schematically depicted in blue in Fig. 1 with the SSBI products indicated in red) is upsampled to $4 \mathrm{Sa}$ /symbol before being processed by the KramersKronig algorithm. From the KK relationship, the optical phase of the transmitted SSB signal is calculated from the detected signal's envelope [3]. By combining the optical phase and amplitude waveforms, a digital representation of the transmitted optical SSB signal is constructed. After downsampling to $2 \mathrm{Sa} / \mathrm{symbol}$, the fiber impairments are compensated using either EDC or DBP. We carried out system simulations, assuming a transceiver with ideal electrical components, i.e. no quantization or other electrical noise, linear IQ modulator and rectangular-shaped OBPF. We simulated the transmission of both single channel $112 \mathrm{~Gb} / \mathrm{s}$ and $50 \mathrm{GHz}$-spaced $9 \times 112 \mathrm{~Gb} / \mathrm{s} \mathrm{WDM}$ 0.51-cycle SSB 16-QAM Nyquist-SCM signals over $160 \mathrm{~km}$ single-span SSMF transmission, with DBP of only the channel of interest. The system parameters are listed in the table in Fig. 1. To assess the impact of the nonlinear distortion on the performance of the KK algorithm, we compared the DBP performance using a KK DD receiver and an ideal linearized receiver, in which the waveform of the transmitted optical signal is input directly to the DBP block (i.e. bypassing the photodetector and the KK block). Single channel and WDM transmission results are plotted 
in Figs. 1(b) and 1(c). It can be seen that at low launch powers, the performance of the KK receiver with DBP is identical to that of the ideal linearized receiver, but, at higher powers, is slightly reduced. In the case of single channel transmission (Fig. 1(b)), the maximum achievable SNR gain with the ideal receiver employing DBP is $5 \mathrm{~dB}$, compared to the EDC case, governed by the nonlinear interaction of signal and ASE noise within the backpropagation. With the KK receiver, an additional penalty arises due to nonlinear distortion reducing the effectiveness of the Kramers-Kronig scheme. The SNR gain achieved by the KK DD receiver with DBP (compared to EDC) was reduced to $3.6 \mathrm{~dB}$. In the case of WDM transmission (Fig. 1(c)), in which the uncompensated interchannel nonlinear effects affect performance, the compensation gains achieved using DBP were $1.1 \mathrm{~dB}$ and $0.7 \mathrm{~dB}$ using the ideal linear receiver and the KK DD receiver respectively.

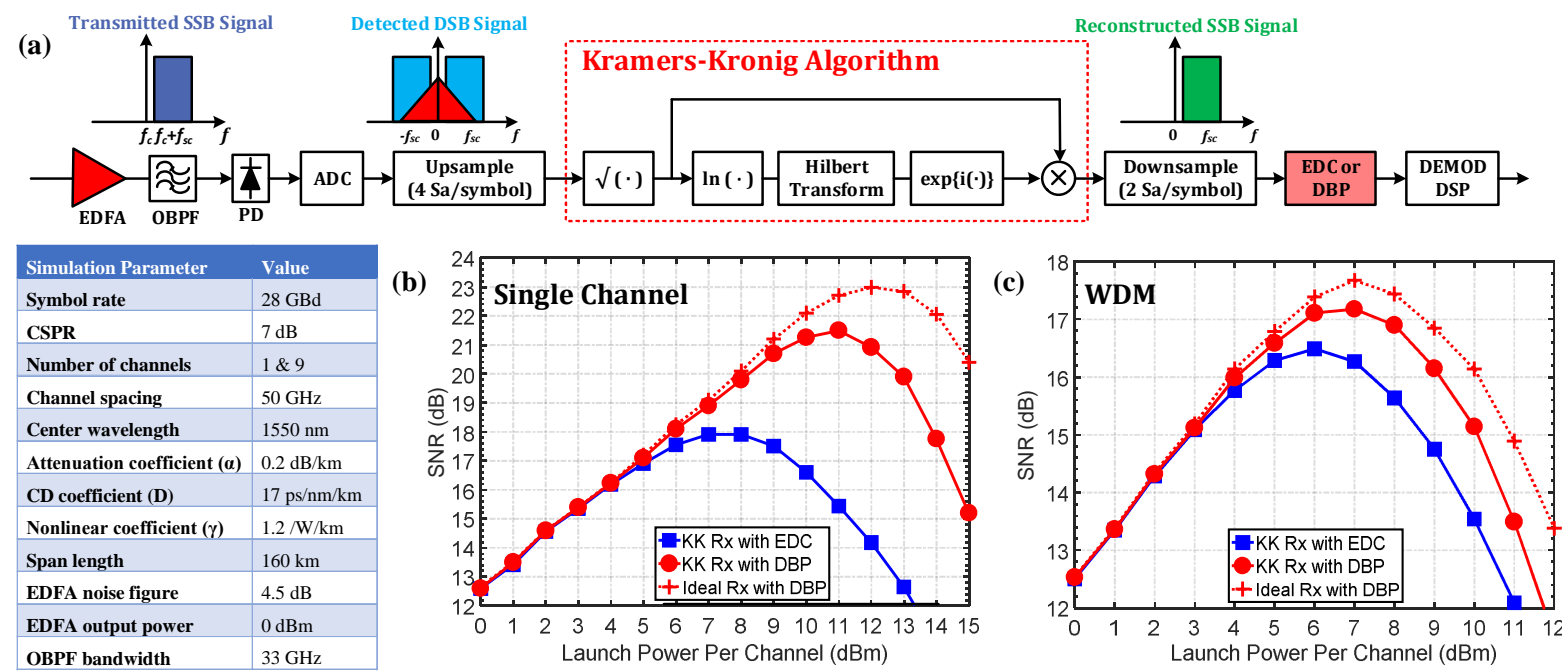

Fig.1: (a) Receiver design including KK scheme combined with EDC or DBP. EDFA: Erbium-doped fiber amplifier. OBPF: Optical band-pass filter. PD: Photodiode. ADC: Analog-to-digital convertor. DEMOD DSP: Demodulation DSP for SSB SCM signal. Simulation results: (b) SNR versus optical launch power at $160 \mathrm{~km}$ single-span single channel transmission. (c) SNR versus optical launch power for $160 \mathrm{~km}$ single-span WDM transmission.

\section{Experimental Demonstration}

The KK DD receiver with DBP was experimentally evaluated using the transmission test-bed shown in Fig. 2. Odd and even channels were separately generated using a pair of IQ-modulators, driven by two 92 GSa/s digital-toanalog convertors (DACs). $28 \mathrm{GBd}(112 \mathrm{~Gb} / \mathrm{s}) 0.51$ cycle SSB 16-QAM Nyquist-SCM signals were generated with a subcarrier frequency of $14.28 \mathrm{GHz}$ and root-raised-cosine filters with a $1 \%$ roll-off factor, and coupled to form a $50 \mathrm{GHz}$-spaced $4 \times 112 \mathrm{~Gb} / \mathrm{s}$ WDM signal (Fig. 2 inset (I)). The optical carrier-to-signal power ratio (CSPR) value was optimized at each distance and launch power to achieve optimum system performance.

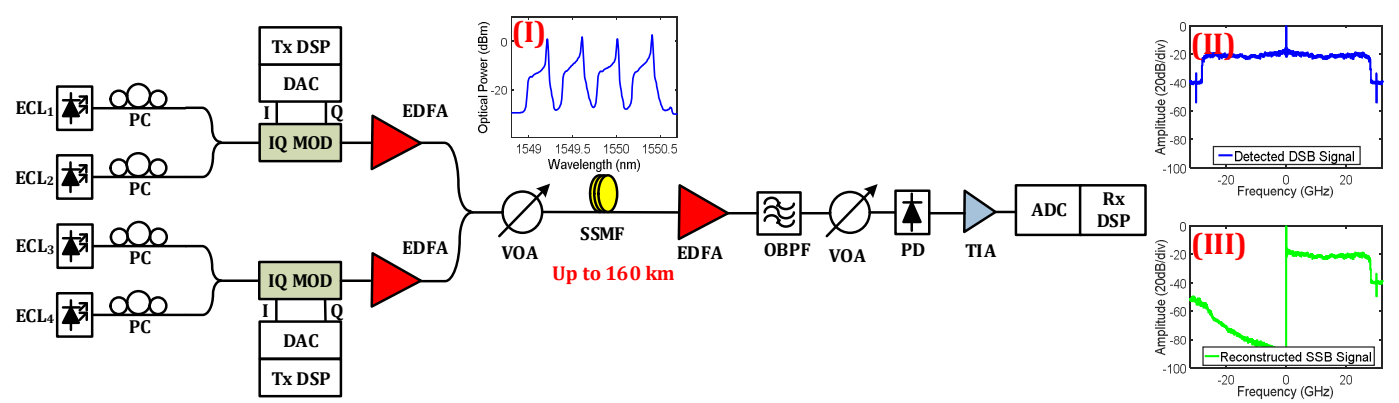

Fig. 2: (a) WDM transmission experimental test-bed. Insets: (I) transmitted WDM spectrum, (II) detected digital DSB signal spectrum and (III) reconstructed digital SSB signal spectrum after the KK scheme. ECL: External cavity laser. PC: Polarization controller. DAC: Digital-to-analog converter. VOA: Variable optical attenuator. SSMF: Standard single-mode fiber.

Transmission experiments were carried out over SSMF links, with span lengths of $80 \mathrm{~km}, 120 \mathrm{~km}$ and $160 \mathrm{~km}$ without inline amplification, followed by an EDFA with a $5 \mathrm{~dB}$ noise figure. At the receiver, a $33 \mathrm{GHz} \mathrm{OBPF}$ was utilized to demultiplex the channel of interest, followed by a $40 \mathrm{GHz}$ bandwidth single PIN photodiode and a single 
$80 \mathrm{GSa} / \mathrm{s}$ analog-to-digital convertor (ADC). The SSBI impairments caused by square-law detection were compensated by the KK scheme, following which, EDC or DBP was used to compensate the fiber impairments (Fig. 1(a)). The DBP was carried out with a total of 10 steps, with step size increasing logarithmically with distance. The digital signal spectra before and after the KK scheme are plotted in Fig. 2 insets (II) and (III).
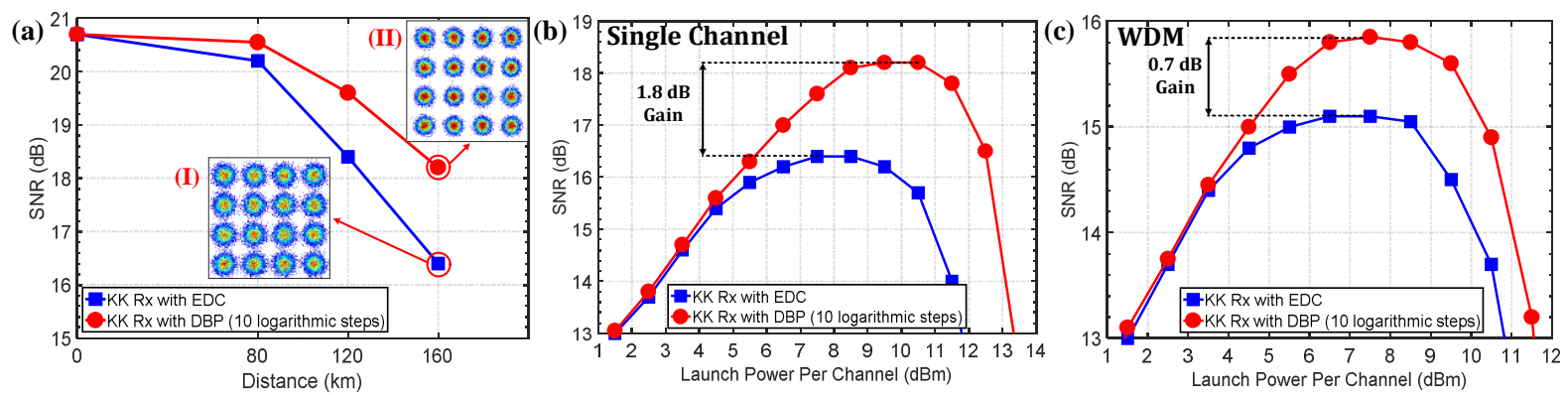

Fig. 3: (a) SNR versus transmission distance in single channel transmission. Insets: Received constellations at $160 \mathrm{~km}$ (I) with $\mathrm{KK}$ and $\mathrm{EDC}(\mathrm{EVM}=16.5 \%)$ and (II) with KK and DBP $(\mathrm{EVM}=12.9 \%)$. (b) Single channel and (c) WDM transmission performance, SNR versus optical launch power per channel at $160 \mathrm{~km}$ transmission distance.

Single channel transmission performance was first assessed (Figs. 3(a) and 3(b)). Fig. 3(a) plots the SNR versus single-span transmission distance. At $80 \mathrm{~km}, 120 \mathrm{~km}$ and $160 \mathrm{~km}$, the received signal-to-noise ratio (SNR) was increased, respectively, from 20.2, 18.4 and $16.4 \mathrm{~dB}$ with EDC to 20.6, 19.6 and $18.2 \mathrm{~dB}$ in the case of DBP. The corresponding compensation gains were found to be $0.4 \mathrm{~dB}, 1.2 \mathrm{~dB}$ and $1.8 \mathrm{~dB}$ and the optimum CSPR values were $12 \mathrm{~dB}, 10 \mathrm{~dB}$ and $8 \mathrm{~dB}$ respectively. The lower DBP gain in the experiment compared to the ideal simulation can be explained by the additional transceiver noise in the experiment, which interacts with the signal during DBP. The effectiveness of the DBP can be observed from the received constellations at $160 \mathrm{~km}$ (Fig. 3 (a) insets (I) and (II)). The minimum BER at this distance was reduced by more than one order magnitude, from $1.4 \times 10^{-3}$ with EDC to $1.3 \times 10^{-4}$ with DBP. SNR versus optical launch power is plotted in Fig. 3(b). Compared to the case with EDC, the optimum launch power increased by approximately $3 \mathrm{~dB}$ with DBP. Finally, WDM transmission over the $160 \mathrm{~km}$ span was evaluated. The SNR versus optical launch power per channel is plotted in Fig. 3(c). Since only the channel of interest was back-propagated, it can be observed that the compensation gain in the WDM case is reduced, due to the additional uncompensated interchannel nonlinear effects. Using DBP, the optimum SNR was increased from $15.1 \mathrm{~dB}$ to $15.8 \mathrm{~dB}\left(0.7 \mathrm{~dB}\right.$ gain), the BER was reduced from $3.8 \times 10^{-3}$ to $1.2 \times 10^{-3}$ and the optimum launch power increased by $0.5 \mathrm{~dB}$.

\section{Conclusions}

We investigated the performance improvement of Kramers-Kronig direct-detection receivers using fiber nonlinearity compensation by digital back-propagation. System performance was assessed by both simulations and experiments in $112 \mathrm{~Gb} / \mathrm{s}$ single channel and $4 \times 112 \mathrm{~Gb} / \mathrm{s}$ WDM SSB 16-QAM Nyquist-SCM DD transmission over single-span SSMF links of up to $160 \mathrm{~km}$. Simulations predict a slight performance degradation of the fiber nonlinearity compensation following the KK algorithm compared to the case of DBP being carried out directly on the received optical signal. However, significant gains were still found to be possible, with experimental confirmation of this. To the best of knowledge, this is the first implementation of the Kramers-Kronig direct-detection receiver with DBP.

This work has been supported by the UK EPSRC UNLOC project.

\section{References}

[1] S. Randel et al., "100-Gb/s discrete-multitone transmission over 80-km SSMF using single-sideband modulation with novel interferencecancellation scheme," Proc. ECOC, Mo.4.5.2 (2015).

[2] A. Mecozzi et al., "Kramers-Kronig coherent receiver," Optica, 3 (11), 1220-1227 (2016).

[3] Z. Li et al., "SSBI mitigation and Kramer-Kronig scheme in single-sideband direct-detection transmission with receiver-based electronic dispersion compensation,” J. Lightw. Technol., 35 (10), 1887-1893 (2017).

[4] X. Chen et al., "218-Gb/s single-wavelength, single-polarization, single-photodiode transmission over 125-km of standard single-mode fiber using Kramers-Kronig detection," Proc. OFC, Th5B.6 (2017).

[5] S. Fan et al., "Twin-SSB direct detection transmission over 80km SSMF using Kramers-Kronig receiver," Proc. ECOC, W.2.D.5 (2017). [6] X. Chen et al., "4×240 Gb/s dense WDM and PDM Kramers-Kronig detection with 125-km SSMF Transmission," Proc. ECOC, W.2.D.4 (2017).

[7] E. Ip et al., “Compensation of dispersion and nonlinear impairments using digital backpropagation,” J. Lightw. Technol., 26 (20), $3416-3425$ (2008).

[8] K. Roberts et al., “Electronic precompensation of optical nonlinearity,” Photon. Technol. Lett., 18 (2), $403-405$ (2006). 